Escuela de Ciencias Sociales y Humanidades, UNED, C.R. URL: http://investiga.uned.ac.cr/revistas/index.php/espiga/index ISSN: 1409-4002 • e-ISSN: 2215-454X

Doi: http://dx.doi.org/10.22458/re.v17i36.2118

\title{
La gestión participativa y sostenible del patrimonio mundial a través de los foros híbridos. El caso del centro histórico de Santa Ana de Cuenca ${ }^{1}$
}

\author{
Bárbara Molina* \\ iD https://orcid.org/0000-0001-5066-1614
}

Recibido: 19 de julio, 2018 - Aceptado: 25 de setiembre, 2018

\section{RESUMEN}

La gobernanza participativa se considera un tema clave en las agendas para el desarrollo sostenible de la época actual. En este contexto, la UNESCO ha buscado incorporar a la sostenibilidad en la gestión de las Ciudades Históricas Patrimonio Mundial. Sin embargo, estos esfuerzos se han visto limitados por la falta de metodologías que permitan la gestión participativa del patrimonio de acuerdo con las características de cada contexto. Por ello, se propone el uso de los denominados foros híbridos como mecanismos de participación, utilizando como caso de estudio

\section{Formato de citación según APA}

Molina, B. (2018). La gestión participativa y sostenible del patrimonio mundial a través de los foros híbridos. El caso del centro histórico de Santa Ana de Cuenca. Revista Espiga, 17 (36), 176-199. Doi: http://dx.doi.org/10.22458/re.v17i36.2118

Formato de citación según Chicago-Deusto

Molina, Bárbara. «La gestión participativa y sostenible del patrimonio mundial a través de los foros híbridos. El caso del centro histórico de Santa Ana de Cuenca». Revista Espiga 17, n. 36 (julio-diciembre, 2018): 176-199. Doi: http://dx.doi.org/10.22458/ re.v17i36.2118

* Doctoranda del programa Sociedad y Cultura: Historia, Antropología, Arte y Patrimonio, Universidad de Barcelona, España. Máster en Gestión del Patrimonio y Museología, Universidad de Barcelona. Licenciatura en Ciencias de la Educación, especialización en Historia y Geografía, Universidad de Cuenca, Ecuador. Correo: baramonei@yahoo.com

1. Por su colaboración en la realización de los foros híbridos, la autora agradece a la Casa de la Cultura Ecuatoriana, especialmente a Martha Orellana, Mónica Muñoz, directora del Museo de las Conceptas, y a la Dra. Ana Luz Borrero, directora de la Cátedra Abierta de Historia de la Universidad de Cuenca. Igualmente agradece al Grupo de Arqueología Pública y Patrimonio de la Universidad de Barcelona, de manera especial a la Dra. Margarita Díaz-Andreu y al Dr. Xavier Roigé i Ventura por su apoyo en la realización del estudio al que se hace mención en este artículo.

Este estudio ha sido financiado por la Secretaría de Educación Superior, Ciencia y Tecnología (SENESCYT), de Ecuador. 
al sitio Patrimonio Mundial de Cuenca (Ecuador). A partir de los datos aquí generados, se busca establecer si esta metodología es efectiva para la gestión sostenible del patrimonio mundial a largo plazo.

Palabras clave: Metodología, foros híbridos, sostenibilidad, gobernanza participativa, patrimonio mundial.

\section{Introducción}

La sostenibilidad, entendida como el desarrollo socioeconómico y medioambiental sin comprometer los recursos de las futuras generaciones, ha sido asumida a nivel mundial, y desde 1992, como modelo para el desarrollo humano². La Organización de las Naciones Unidas para la Educación, la Ciencia y la Cultura (UNESCO), como ente cultural adscrito a la Organización de las Naciones Unidas (ONU), ha buscado incorporarse a este modelo al realizar, en los dos últimos decenios, un esfuerzo para sumar sus preceptos en los sistemas de gestión de los Sitios Patrimonio Mundial. Para hacer esto posible, sin embargo, una de las necesidades identificadas por esta entidad ha sido la de contar con metodologías que permitan tal incorporación. Esta carencia metodológica, como se plantea en este artículo, ha provocado que desde el ámbito de los estudios patrimoniales se trabaje en la importancia de la inclusión de los diversos actores como piedra angular de los procesos de gestión sostenible del patrimonio mundial a través de la gobernanza participativa ${ }^{3}$.

La gobernanza participativa, en el contexto de la sostenibilidad, ha tomado relevancia porque se la entiende como una práctica deliberativa, democrática e inclusiva para la formulación de políticas, diseño de alternativas y opciones de inversión, gestión y monitoreo del desarrollo de intervenciones en las comunidades ${ }^{4}$. Se ha escrito mucho sobre la gobernanza participativa a través de la gestión del patrimonio, tanto por parte

2. Gro Harlem Brundtland, «Our common future» (Report of the World Commission on environment and development: United Nations, 1987). Amartya Sen, «Development as capability expansion» (conferencia, Universidad de Oxford, 1990). ONU, Declaración de Río sobre el Medioambiente y el Desarrollo, 1992.

3. Ver: UNESCO, Convención de Patrimonio Mundial, 1972; Convención para la salvaguardia del patrimonio cultural inmaterial, 2003; Convención sobre Protección y Promoción de la Diversidad de las Expresiones Culturales, 2005; Recomendación sobre el paisaje urbano histórico, 2011; Consejo de Europa, Serie de Tratados del Consejo de Europa n. ${ }^{\circ}$ 199, 2005. Simon Makuvaza, «The Management of Cultural World Heritage Sites and Development in Africa: History, Nomination Processes and Representation on the World Heritage List» (Springer Science \& Business Media, 2014). Sofia Labadi y William Logan, «Urban heritage, development and sustainability» (Londres/New York, Routledge, 2015). Amareswar Galla, World Heritage: Benefits beyond Borders (Cambridge University Press, 2012). Chiristine Landorf, "Governance in historic urban environments: A theoretical review», International Journal of Heritage and Sustainable Development, 1(1) (2011): 7-16. Ana Pereira Roders y Ron van Oers «World Heritage Cities Management», Facilities 29, n. ${ }^{\circ} 7 / 8$ (2011): 276-85.

4. The World Bank, «Participatory development and the World Bank: Potential directions for change» (artículo de discusión número 183, Washington DC, 1992). David Wilcox, «The guide to effective participation» (Delta Press, 1994). Elizabeth M. Rocha, «A Ladder of Empowerment», Journal of Planning Education and Research 17, n. ${ }^{\circ} 1$ (septiembre 25, 1997): 31-44. 
de la UNESCO como de otros profesionales del patrimonio, con un énfasis en cuanto a las diversas razones que justifican la necesidad de su aplicación. A pesar de ello, en realidad son escasas las publicaciones sobre los ejemplos prácticos relativos a la gestión participativa de los Sitios Patrimonio Mundial y, desde este punto de vista, es importante reflexionar sobre los foros híbridos propuestos por Michael Callon, Pierre Lascoumes y Yannick Barthe ${ }^{5}$. Estos, se definen como dispositivos de consulta con un enfoque de abajo hacia arriba, donde una serie de participantes heterogéneos pueden llegar a tomar decisiones de manera abierta y sin jerarquías a partir de la controversia. El fin último de los foros híbridos es generar lo que los autores ${ }^{6}$ denominan democracia dialógica, entendida como el derecho social de construir un mundo común a través del diálogo entre todas las identidades y colectividades; en otras palabras, la gobernanza participativa.

La propuesta presentada aquí apuesta por el uso de los foros híbridos como método que contribuya a las limitadas opciones metodológicas de gestión participativa en las Ciudades Históricas Patrimonio Mundial. Estos foros tienen la finalidad de explorar las relaciones entre los diversos actores y el patrimonio, así como empoderarlos en el contexto de la gobernanza participativa del patrimonio. Para ello, y a manera de marco teórico, en este artículo se analiza en primer lugar las relaciones entre la sostenibilidad, la gobernanza y el patrimonio mundial, sobre la base de la literatura académica. A continuación, y con fundamento en el trabajo de Callon et al. ${ }^{7}$ y de Rodney Harrison ${ }^{8}$, el artículo presenta el proceso de planeamiento y ejecución de cuatro foros híbridos en el Sitio Patrimonio Mundial de Santa Ana de Cuenca, en Ecuador. Finalmente, este trabajo ofrece una discusión metodológica de los resultados de los foros híbridos con el objetivo de aportar argumentos sobre la validez de estos mecanismos de participación como herramientas para la gestión participativa del patrimonio mundial a largo plazo.

\section{Gobernanza participativa, sostenibilidad y patrimonio mundial}

\section{Gobernanza participativa y sostenibilidad}

La gobernanza participativa es una rama de la teoría de la gobernanza que hace hincapié en la participación democrática mediante prácticas deliberativas, opuestas a la visión tradicional que generalmente dificulta la realización de una democracia participativa genuina ${ }^{9}$. La gobernanza participativa toma fuerza en la década de $1990^{10}$, especialmente influenciada por la teoría de las capacidades de Amartya Sen. Este autor plantea que los gobiernos deberían ser juzgados en función de las capacidades reales que tienen

5. Michael Callon, Pierre Lascoumes y Yannick Barthe, «Acting in an Uncertain World: An Essay on Technical Democracy», 2009.

6. Ibíd.

7. Ibíd.

8. Rodney Harrison, Heritage: Critical Approaches (Routledge, 2013).

9. Mark Bevir, A theory of governance (Berkeley/Los Angeles, California: University of California Press, 2013).

10. Frank Fisher, «Participatory Governance: From Theory to Practice» en The Oxford Handbook of Governance, ed. por David Levi-Faur (Oxford: Oxford University Press, 2012), 457-71. Landorf, «Governance in Historic...». 
los gobernados para ejercer plenamente su derecho a la libertad. De acuerdo con los preceptos de la gobernanza participativa, la gente, especialmente aquellos en desventaja, puede contribuir a la formulación de políticas, diseño de alternativas y opciones de inversión, gestión y monitoreo del desarrollo de intervenciones en las comunidades ${ }^{11}$.

Tanto la Conferencia sobre el Medioambiente y el Desarrollo, a través de sus Objetivos de Desarrollo Sostenible ${ }^{12}$, como la Agenda 21 para el Desarrollo ${ }^{13}$, ofrecen un enfoque claro sobre la participación como la piedra angular que ha de llevar a la sostenibilidad $^{14}$. En este contexto, existe un consenso entre los profesionales y las diversas instituciones para el desarrollo en cuanto a las ventajas que en teoría supone la gobernanza participativa para la sostenibilidad ${ }^{15}$. Por una parte, esta permite conocer las necesidades y motivaciones de los diversos actores ${ }^{16}$; por otra, facilita la distribución equitativa y justa del poder político, los recursos y los beneficios entre todos los implicados ${ }^{17}$. La gobernanza participativa puede ofrecer gran flexibilidad en la resolución de problemas y el uso efectivo de recursos ${ }^{18}$, al desarrollar la comunicación a través de la mejora en los flujos de información entre los actores y el gobierno, a la vez que fomenta las capacidades de la comunidad ${ }^{19}$. Mejorar las capacidades comunitarias permite la expansión de la infraestructura pública y una mayor eficiencia en los servicios ${ }^{20}$, lo que reduce las decisiones discrecionales, estableciendo una responsabilidad compartida basada en la confianza y la reciprocidad ${ }^{21}$. Esto exige un cambio de gobierno a gobernanza que se logra empoderando a los diversos actores (ciudadanos, profesionales, gestores, empresarios) a

11. Michela Arnaboldi y Nicola Spiller, «Actor-Network Theory and Stakeholder Collaboration: The Case of Cultural Districts», Tourism Management 32, n. ${ }^{\circ} 3$ (2011): 641-54.Sherry R. Arnstein, "A Ladder Of Citizen Participation», Journal of the American Institute of Planners 35, n. ${ }^{\circ} 4$ (julio 1969): 216-24. Sen, «Development as capability.....The World Bank, «Participatory development.., 2. Wilcox, «The guide to effective participation». Rocha, «A Ladder of Empowerment».

12. PNUD, Agenda para el Desarrollo Sostenible 2030 (PNUD, 2016).methodologylity, participatory governance, world heritagenio mundial.sos actores y el patirmonioor) sostenible de nuestro tiempomethodologylity, participatory governance, world heritagenio mundial.sos actores y el patirmonioor) sostenible de nuestro tiempo

13. ONU, Agenda 21, Programa de Acción las Naciones Unidas. New York: UN Publications, 1992.

14. Bárbara Molina, «La incorporación de la cultura y el patrimonio en el desarrollo sostenible: desafíos y posibilidades», Revista Humanidades, 8(1), 2018. PNUD, «Agenda para el Desarrollo...».

15. Johanna Speer, «Participatory Governance Reform: A Good Strategy for Increasing Government Responsiveness and Improving Public Services?», World Development 40, n. ${ }^{\circ} 12$ (2012): 2379-98. Hartmut Schneider, «Participatory Governance for Poverty Reduction», Journal of International Development 11, n. 4 (junio 1999): 521-34. Benjamín Goldfrank, «Lessons from Latin America's experience with participatory budgeting», en Participatory budgeting, ed. por Awar Shah (Washington, DC: World Bank, 2007): 91-126.

16. Mark E. Warren, «Institutionalizing Deliberative Democracy», en Deliberation, Participation and Democracy (London: Palgrave Macmillan UK, 2007): 272-88.

17. Speer, «Participatory Governance...».

18. Esther H.k. Yung y Edwin H.w. Chan, «Problem Issues of Public Participation in Built-Heritage Conservation: Two Controversial Cases in Hong Kong Habitat International» 35, n. 3 (2011): 457-66.

19. Fisher, «Participatory governance...».

20. Peter Evans, «Development as Institutional Change: The Pitfalls of Monocropping and the Potentials of Deliberation», Studies in Comparative International Development 38, n. 4 (December 2004): 30-52.

21. Fisher, «Participatory governance...». 
través de la participación ${ }^{22}$. Para ello, es necesario redistribuir la capacidad de decisión lejos de estructuras centralizadas y jerárquicas hacia otras con un enfoque más localizado y colectivo ${ }^{23}$ a través del trabajo conjunto de los actores organizados en redes locales y comunitarias $^{24}$. La necesidad de idear formas para hacer posible que las personas se impliquen en la gobernanza del patrimonio, responde al derecho que estas tienen de decidir sobre cuestiones que les afectan. Más aún en el contexto actual, en el que los gobiernos insisten en la sostenibilidad como un aspecto clave y que, por tanto, debería reflejarse en políticas y programas a todos los niveles ${ }^{25}$. Los proyectos de presupuestos participativos de Porto Alegre (Brasil) y Montevideo (Uruguay) ${ }^{26}$, y el planeamiento colaborativo de infraestructuras y planes de gestión en Medellín (Colombia) y Kerala (India), son ejemplos de cómo los ciudadanos pueden, dadas las condiciones adecuadas, participar con altos niveles de competencia al momento de establecer cómo y en qué se ha de invertir el dinero público ${ }^{27}$. Sin embargo, la réplica positiva de estas metodologías resulta compleja ante las múltiples variables que son únicas de cada contexto ${ }^{28}$. Por tanto, la gobernanza participativa plantea la necesidad de realizar un análisis de las relaciones que existen entre los diversos actores y su contexto para entender la forma en la cual se distribuye el poder entre ellos ${ }^{29}$.

22. Hartmut Bossel, Indicators for Sustainable Development: Theory, Method, Applications (International Institute for Sustainable Development Winnipeg, 1999). Ignacio Farías, «Devising Hybrid Forums: Technical Democracy in a Dangerous World», City 20, n. ${ }^{\circ} 4$ (2016): 549-62. Harrison, Heritage: Critical Approaches. Landorf, «Governance in Historic...».

23. Landorf, «Governance in historic...».

24. Paul Williams, «The Governance of Sustainable Development in Wales», Local Environment 11, n. 3 (junio 2006): 253-67. Bruno Latour, «Reassembling the Social», Hampshire: Oxford University Press, 2007. Arnaboldi y Spiller, «Actor-network theory... Arnstein, «A ladder of citizen...». Harrison, Heritage: critical approaches. Landorf, «Governance in Historic...».

25. Vasudha Chhotray y Gerry Stoker, Governance Theory and Practice: A Cross Disciplinary Approach (Basingstoke, Palgrave Macmillan, 2009). Nick Gallent, «Strategic-Local Tensions and the Spatial Planning Approach in England», Planning Theory \& Practice 9, n. 3 (septiembre 2008): 307-23. Landorf, «Governance in Historic... ». Molina «La incorporación de la cultura...».

26. Benjamin Goldfrank, "The Fragile Flower of Local Democracy: A Case Study of Decentralization/ Participation in Montevideo», Politics \& Society 30, n. ${ }^{\circ} 1$ (marzo 17, 2002): 51-83. Benjamín Goldfrank, «Lessons from Latin...».

27. Speer, «Participatory Governance...».

28. Benjamin. Goldfrank, Deepening Local Democracy in Latin America : Participation, Decentralization, and the Left (The Pennsylvania State University Press, 2011).

29. Yulong Li y Caroline Hunter, «Community Involvement for Sustainable Heritage Tourism: A Conceptual Model», Journal of Cultural Heritage Management and Sustainable Development 5, n. ${ }^{\circ} 3$ (2015): 24862. Laura J. Lawton y David B. Weaver, «Using Residents’ Perceptions Research to Inform Planning and Management for Sustainable Tourism: A Study of the Gold Coast Schoolies Week, a Contentious Tourism Event», Journal of Sustainable Tourism 23, n. ${ }^{\circ} 5$ (mayo 28, 2015): 660-82. Arnstein, "A ladder of citizen...». Callon et at., Acting in an uncertain world... Fisher, «Participatory Governance...». Harrison, Heritage: Critical Approches... 
La literatura actual sobre gestión patrimonial establece que el entendimiento holístico del patrimonio está determinado por una participación diversa en la gestión ${ }^{30}$. Sin embargo, el intento de la gestión patrimonial de las ciudades históricas por acoplarse a los principios de la gobernanza participativa y de la sostenibilidad ha sido difícil. Esto principalmente porque su ejecución se encuentra integrada en la práctica a diversas cuestiones socioculturales, políticas, económicas y medioambientales que convierte a la gestión de estas ciudades en un ejercicio amplio y complicado ${ }^{31}$. Esta complejidad dificulta la transferibilidad de las propuestas realizadas a nivel teórico desde las instituciones patrimoniales como la $\mathrm{UNESCO}^{32}$. Al tratarse de conceptos y metodologías propuestos desde una visión generalizada internacional, la efectiva integración de estos en la práctica dificulta la gestión y, en consecuencia, la gobernanza de estos Sitios Patrimonio Mundial ${ }^{33}$. A esto se suman otros problemas como la débil vinculación entre lo histórico y las identidades contemporáneas y el privilegio de la identidad nacional sobre la local y sus múltiples experiencias ${ }^{34}$.

En lo que respecta a los instrumentos de gestión, el principal problema que se ha identificado para la gestión participativa de las Ciudades Históricas Patrimonio Mundial, ha sido la falta de experiencia y de posibilidades en la ejecución de los planes de manejo ${ }^{35}$. Esto, sobretodo, porque la pertinencia de estas herramientas sigue siendo objeto de debate entre los profesionales del patrimonio ${ }^{36}$, principalmente por su dificultad de integración con otros instrumentos legislativos y de gestión existentes y por la complejidad que entraña incluir a diversos participantes en su diseño y ejecución. Adicionalmente, la efectiva aplicación de los planes de gestión en las ciudades históricas patrimoniales, depende de factores como la estabilidad política, la transparencia, la capacidad técnica, científica, de financiamiento y, como ya se ha mencionado en el párrafo anterior, las

30. Matthias Ripp y Dennis Rodwell, «Governance in UNESCO World Heritage Sites: Reframing the Role of Management Plans as a Tool to Improve Community Engagement», en Aspects of Management Planning for Cultural World Heritage Sites (Cham: Springer International Publishing, 2018): 241-53. Vasco Monteiro, Marco Painho y Eric Vaz, «Is the Heritage Really Important? A Theoretical Framework for Heritage Reputation Using Citizen Sensing», Habitat International 45 (2015): 156-62. Rob Pickard, «Management strategies for historic towns in Europe», en Urban heritage, development and sustainability: International frameworks, national and local governance, ed. Sofia Labadi (London/New York: Routledge, 2016): 151-174. Labadi y Logan, «Urban heritage...».

31. Fernando Carrión, «Centro Histórico: La polisemia del espacio público», acceso: 07 de octubre, 2018, http://www.redalyc.org/pdf/1151/115112535008.pdf

32. Katriina Soini y Joost Dessein, «Culture-Sustainability Relation: Towards a Conceptual Framework», Sustainability 8, n. 2 (febrero 11, 2016): 167. Simon Makuvaza, Aspects of Management Planning for Cultural World Heritage Sites: Principles, Approaches and Practices (Springer, 2017). Ripp y Rodwell, «Governance in UNESCO...».

33. Antonios Vlassis, «Culture in the Post-2015 Development Agenda: The Anatomy of an International Mobilisation», Third World Quarterly 36, n. ${ }^{\circ} 9$ (septiembre 2, 2015): 1649-62. Molina, «La incorporación de la cultura...». Bandarin y Van Oers, Reconnecting the city...

34. Laurajane Smith, Uses of Heritage (Routledge, 2006). Harrison, Heritage: Critical Approaches. Landorf, «Governance in Historic...». Ripp y Rodwell, «Governance in UNESCO...». Labadi y Logan, «Urban Heritage...».

35. Ripp y Rodwell, «Governance in...».

36. Francesco. Bandarin, Ron van Oers, Reconnecting the City... 
demás características del contexto. Por lo tanto, en la planificación sostenible, la clave para la gestión de las Ciudades Históricas Patrimonio Mundial, no está en el supuesto teórico de incluir a los diversos actores en el diseño de objetivos, implementación y análisis de resultados y medidas correctivas ${ }^{37}$. La clave está en cómo hacerlo bajo las circunstancias reales de cada caso. De ahí la importancia de todos los actores, pues son ellos los que informan sobre las características propias del contexto ${ }^{38}$.

\section{Foros híbridos para la exploración de controversias, el empoderamiento y la gestión participativa de los Sitios Patrimonio Mundial}

\section{Foros híbridos como metodología participativa}

Los foros híbridos surgen de la sociología constructivista como una propuesta teórica para evaluar las asimetrías entre expertos técnicos, científicos y legos ${ }^{39}$. Se fundamentan, por una parte, en la teoría actor-red desarrollada principalmente por Michael Callon y Bruno Latour ${ }^{40}$; esta supone que los fenómenos han de analizarse en función de las interacciones entre todos los componentes de una red, referidos estos como actantes; es decir, elementos humanos y no humanos en constante interacción. Por otra parte, en las propuestas de la evaluación constructivista, que privilegia el papel activo de los actores como creadores de significados ante circunstancias reales que requieren el uso de habilidades, capacidades y conocimientos para generar un conocimiento colaborativo basado en la realimentación, la reflexión y la autoevaluación ${ }^{41}$.

El foro híbrido se plantea como un procedimiento de consulta que establece una dinámica de exploración para la búsqueda de escenarios comunes entre los diversos actores, rompiendo las asimetrías de poder y estatus entre ellos ${ }^{42}$. Esto exige el desarrollo de una controversia organizada y transparente en la que cada participante aporta su propia visión y conocimientos sobre la cuestión. Por esta razón, el carácter híbrido de los foros radica en que sus representantes son heterogéneos y, por tanto, las cuestiones y controversias se tratan a diferentes niveles y desde una variedad de puntos de vista ${ }^{43}$. Se parte de la base de que cada actor tiene un conocimiento parcial y limitado sobre la cuestión en discusión y de ahí que uno de los objetivos principales del foro híbrido es que el grupo llegue a conocer, con más profundidad, el conjunto de problemas relevantes que afectan al fenómeno en discusión. Con este sistema se pretende superar lo que los

37. UNESCO, Operational Guidelines for the Implementation of the World Heritage Convention, 2017, 119: 31-32.

38. Patrick M. Condon, Design Charrettes for Sustainable Communities (Island Press, 2012). Ripp y Rodwell, "Governance...». Callon et al., Acting in an Uncertain World.

39. Paloma Díaz García, «El principio de precaución como base de los foros híbridos», Ética en la práctica, (Granada: Editorial Universidad de Granada, 2008): 259-278.

40. Bruno Latour-Soziale welt, «On Actor-Network Theory. A Few Clarifications plus More than a Few Complications», 1996, Bruno-Latour.Fr, acceso: 07 de octubre, 2018, http://www.bruno-latour.fr/sites/default/files/P-67 ACTOR-NETWORK.pdf

41. Alfonso Gonzáles, «El turismo desde un enfoque de sociología constructivista», Teoría y praxis, 5-6 (2009):109-24. Paloma Díaz García, «El principio de...».

42. Callon et al., Acting in an Uncertain World. Farías, «Devising hybrid forums...».

43. Callon et al., Acting in an Uncertain World, 18. 
autores consideran los desafíos democráticos que provienen de la imposición política de soluciones avaladas por criterios técnicos y científicos sin incluir a los demás integrantes de la sociedad. De este modo se alcanza, según Callon et al., la democracia dialógica.

Para conseguir una genuina práctica dialógica ${ }^{44}$, el foro híbrido debe cumplir con una serie de principios. El primero es el de controversia, que refiere a la discusión entre diferentes actores que mantienen posturas contrapuestas y un conocimiento parcial, esto lleva al foro híbrido a generar posturas nuevas y colectivas con base en la negociación. El principio de incertidumbre implica una proyección de futuro que exige considerar todos los posibles escenarios para definir cualquier acción venidera. Estos escenarios no pueden proponerse desde un solo punto de vista, sino de varios, ya que pone en duda lo que cada uno de los participantes considera como verdad. El tercer y último principio, propuesto por Callon, Lascoumes y Barthe ${ }^{45}$, es el denominado de precaución, que reside en la postura cautelar necesaria en la búsqueda de soluciones, ya que se considera necesario conocer y evaluar todos los componentes de la cuestión a debate (especialmente la diversidad de posturas y enfoques) antes de considerar una solución como definitiva. Callon et al. ${ }^{46}$ sostienen que, para garantizar la consecución de estos principios, es necesaria la continuidad de los procesos. Sin embargo, en este artículo se propone a la continuidad no como un medio, sino como un principio, entendido este como el requisito clave que prolonga el diálogo a partir del desarrollo de las relaciones con los ahora nuevos socios cuando las circunstancias lo requieran.

\section{Foros híbridos en la gestión participativa del patrimonio}

Desde finales de la década de 1980, especialmente, el planteamiento teórico de los foros híbridos como mecanismos de participación ha sido originalmente vinculado a temas medioambientales y de salud pública. La incursión de la teoría de los foros híbridos en los estudios patrimoniales solo fue propuesta hace un lustro por Rodney Harrison ${ }^{47}$, quien siguiendo de cerca los postulados de Callon et al. ${ }^{48}$ propuso de manera teórica la inclusión de esta metodología en la gestión del patrimonio como un mecanismo válido para conseguir una relación dialógica entre el patrimonio, expertos, ciudadanos ordinarios y la sostenibilidad ${ }^{49}$. Del mismo modo, y con base en el trabajo de los autores previamente mencionados, en este texto se propone el uso de los foros híbridos como método de exploración, participación y empoderamiento para la gestión sostenible de las Ciudades Históricas Patrimonio Mundial. En primer lugar como método de exploración porque permite la identificación de las identidades de los diversos actores, su posición en términos políticos, económicos y culturales, las preocupaciones, necesidades y tensiones que manejan en relación con el patrimonio y su nivel de empoderamiento ${ }^{50}$. Los foros híbridos, por otra parte, son instrumentos de participación porque asumen que los fenómenos

\footnotetext{
44. Ibíd.

45. Callon et al., Acting in an Uncertain World.

46. Ibíd.

47. Harrison, Heritage: Critical Approaches.

48. Callon et al., Acting in an Uncertain World.

49. Harrison, Heritage: Critical Approaches, 204-205.

50. Callon et al., Acting in an Uncertain World, 180. Harrison, Heritage: Critical Approaches, 225.
} 
no se producen de forma aislada, sino que al propiciar un diálogo continuo y oportuno con nuevos actores e identidades, reduce jerarquías, divisiones y resentimientos entre los participantes ${ }^{51}$. Finalmente, los foros híbridos son un método de empoderamiento porque ayudan, a través de la consulta, al ejercicio de la democracia participativa, ya que se apoyan en una intensa circulación de información que favorece la construcción de un conocimiento colaborativo de tipo científico, profesional y civil ${ }^{52}$. Este conocimiento se debe construir desde horizontes epistemológicos diversos, al combinar datos teóricos con observaciones empíricas, datos objetivos y subjetivos ${ }^{53}$, lo que exige un análisis atento del contexto local desde diversas posturas, percepciones y necesidades.

\section{Foros híbridos como herramientas de exploración y empoderamiento en el centro histórico de Santa Ana de Cuenca}

Como se ha mencionado en párrafos anteriores, es escasa la literatura sobre la aplicación práctica de la metodología de los foros híbridos en general y en particular en el contexto del patrimonio. Esta situación llevó a plantear la aplicación de los supuestos teóricos relativos a los foros híbridos en el contexto de las dinámicas entre el patrimonio y la comunidad, para de este modo entender mejor sus relaciones. Para ello, se tomó como caso de estudio al Centro Histórico Patrimonio Mundial de Santa Ana de Cuenca, en Ecuador, en el cual se ejecutaron cuatro foros híbridos, estableciendo como punto de partida la exploración de las diversas posturas que se han generado en torno a la construcción de un tranvía. En este apartado se expondrá cómo se materializaron en la práctica los conceptos y la teoría relativa a los foros híbridos desde febrero de 2016 hasta abril de 2017. Con esta información se pretende aportar argumentos que validen a los foros híbridos como mecanismos de participación en general y, en particular, si su aplicación en el contexto local tiene posibilidades de convertirse en una buena metodología para la gestión participativa del Sitio Patrimonio Mundial de Santa Ana de Cuenca a largo plazo. Cabe mencionar que en este trabajo no se pretende analizar los resultados obtenidos en dichos foros, lo que será objeto de un artículo diferente, sino centrarse en la metodología seguida para que esta experiencia pueda servir como guía a otras que pretendan utilizarla.

\section{El centro histórico de Santa Ana de Cuenca como Patrimonio Mundial}

La ciudad de Santa Ana de Cuenca se ubica al sur de los andes ecuatorianos, en un valle a $2560 \mathrm{~m} \mathrm{~s}$. n. m. Su centro histórico fue declarado patrimonio mundial por la UNESCO en diciembre de 1999, de acuerdo con los criterios II, IV y V que hacen referencia a su excepcional traza urbana colonial de acuerdo con el modelo renacentista de Carlos V, su interesante mestizaje arquitectónico y por ser un ejemplo único de ciudad agrícola de fundación colonial en valle montañoso ${ }^{54}$.

\footnotetext{
51. Callon et al., Acting in an Uncertain World, 221.

52. Ibíd, 201.

53. Ibíd.

54. Mayor información en: Historic Center of Santa Ana de Cuenca, acceso: 07 de octubre, 2018, http://whc. unesco.org/en/list/863
} 
«La inclusión de Cuenca en la Lista de Patrimonio Mundial fue resultado de una hábil maniobra política basada en el aprovechamiento de diferentes coyunturas políticas ${ }^{55}$. Al momento de la declaratoria, el centro histórico no contaba con un plan de gestión, a pesar de que este ya se consideraba como requisito ${ }^{56}$. Desde entonces, 18 años después, aun no ha sido posible concretarlo ${ }^{57}$. Desde el 2005, y de acuerdo con las directrices prácticas de la UNESCO, los planes de gestión obligatoriamente han de incluir la participación de la comunidad en la gestión patrimonial, lo que está relacionado con el paradigma de sostenibilidad ${ }^{58}$. Para el caso de Cuenca, los foros híbridos se presentan aquí como una opción para implementar esta normativa de la UNESCO y permitir la participación de la comunidad en la gestión sostenible del patrimonio, siempre desde el análisis del contexto.

El proceso de realización de los foros híbridos ha permitido, en primer lugar, establecer los criterios y recursos necesarios para su realización, identificar las dificultades reales que involucra su práctica y, finalmente, ha permitido contrastar las diferentes circunstancias en las que se llevó a cabo cada foro, para de este modo analizar cuáles son las posibilidades que estos pueden tener como instrumentos para la gestión participativa del Centro Histórico Patrimonio Mundial de Cuenca.

\section{Ejecución de los foros híbridos en el centro histórico Patrimonio Mundial de Santa Ana de Cuenca}

Para realizar los foros híbridos en la ciudad de Cuenca bajo la lógica de los principios presentados en el apartado denominado Foros híbridos para la exploración de controversias, el empoderamiento y la gestión participativa de los Sitios Patrimonio Mundial, fueron necesarias tres fases: planeamiento, preparación y ejecución. La primera, realizada entre febrero 2016 y marzo de 2017, consistió en recabar información sobre el contexto local en cuanto este resulta de una historia, cultura, legislación y trayectorias políticas únicas ${ }^{59}$. Durante esta fase se consultó el marco legal vigente en temas de patrimonio, uso del espacio público y participación. Igualmente se solicitaron diversos documentos a las diferentes instituciones con atribuciones en la gestión del patrimonio y la cultura. De especial importancia fueron los presupuestos, planes operativos anuales, informes de rendición de cuentas, de socialización de proyectos, participación y vinculación con la comunidad, además de convenios y programas de colaboración institucional y comunicación. Estos documentos son importantes en cuanto informan sobre el estado actual de la cuestión en lo referente a existencia de redes y la gestión participativa del patrimonio. Tras recopilar esta información, a continuación se creó una base de datos de actores e

55. Fausto Cardoso, correo electrónico al autor, 12 de julio de 2018.

56. Birgitta Ringbeck, «Management Plans for World Heritage Sites», A Practical Guide, 2008.

57. Rey Pérez, Julia et al., Paisaje urbano histórico. La aplicación de la recomendación sobre el paisaje urbano histórico (PUH) en Cuenca (Ecuador). Una nueva aproximación al patrimonio cultural y natural. Universidad de Cuenca, Cuenca, 2017.

58. UNESCO, «Directrices Prácticas...», 119.

59. Francesco Bandarin y Ron van Oers, eds., Reconnecting the City (Oxford, UK: John Wiley \& Sons, Ltd., 2014). 
instituciones para identificar la existencia y nivel de entramado de las redes comunitarias, sociales, profesionales y comerciales entre otras, ya que esto se hace necesario en la movilización de una amplia variedad de público con diversos conocimientos y más allá de los representantes delegados ${ }^{60}$. Gracias a esto, fue posible identificar y estimar los recursos económicos, físicos, técnicos y humanos necesarios para la producción de los foros híbridos. Este punto fue clave en cuanto varios actores interesados ofrecieron sus canales de difusión, apoyo logístico e infraestructuras físicas para la realización abierta y habilitada de los foros híbridos. Una vez identificados los recursos disponibles, se desarrolló la fase de preparación entre febrero y marzo de 2017. Con base en la investigación previa del contexto, se elaboró un plan de ejecución que consistió en determinar la información y contenidos a discutir, preparar el espacio físico y los recursos técnicos para la acogida de los participantes. Parte importante en esta fase fue el trabajo con el equipo humano, especialmente con un moderador con conocimientos en mediación, con el que se elaboraron unas «reglas del juego ${ }^{61}$ a manera de código deontológico para garantizar la libertad de expresión de todos los participantes de manera organizada. El último paso dentro de la fase de preparación fue la difusión del foro híbrido por diversos medios de comunicación, especialmente institucionales (cuando existieron), redes sociales y a través de la prensa local, hecho bastante sorpresivo porque fueron los comunicadores sociales quienes ofrecieron estos canales de difusión en todos los casos.

Los principales inconvenientes encontrados en estas primeras fases fueron, por una parte, la escasa existencia de redes locales, tanto a nivel institucional como de actores externos, lo que ralentizó el contacto con los diversos implicados tanto en temas de patrimonio como de sostenibilidad, lo que evidenció una mínima relación entre ellos. Por otra parte, el acceso a la documentación pública, principalmente en las dependencias del Gobierno Autónomo Descentralizado (GAD) de Cuenca, como ente responsable de la gestión directa del conjunto patrimonial, por la ausencia de un sistema organizado de archivos.

Luego de la fase de preparación siguió la de ejecución, que se llevó a cabo a finales de marzo y a lo largo de abril de 2017, cuando se realizaron un total de cuatro foros híbridos en diferentes espacios (Tabla 1).

Como se verá más adelante, la multiplicidad de espacios permitió comprobar que este aspecto es determinante en la ejecución y los resultados del foro híbrido. Una dificultad práctica que se tuvo que afrontar durante la realización de los foros híbridos, fue el tiempo de duración, el cual no está estipulado en la literatura académica. En este caso, y con base en la experiencia del primer foro realizado que resultó demasiado largo, se considera necesario un promedio de tres horas para los demás. En cuanto a la ejecución del foro híbrido, y como propone Farías ${ }^{62}$, los primeros minutos se destinaron a entregar información clara, accesible y objetiva sobre las reglas y objetivos del proceso, así como del tema a partir del cual se generaría la controversia. En segundo lugar, que los participantes iniciaran el debate con algo de información, pero siendo conscientes de que la

60. Ignacio Farías, «Devising Hybrid Forums...».

61. Ibíd.

62. Ibid. 
TABLA 1

Descripción de las condiciones para la ejecución de los diferentes foros híbridos

\begin{tabular}{|c|c|c|c|c|c|c|c|}
\hline \multirow[t]{2}{*}{ Foro } & \multirow[t]{2}{*}{ Temática } & \multirow[t]{2}{*}{ Lugar } & \multirow{2}{*}{$\begin{array}{c}\text { Día y hora } \\
\text { de ejecución (2017) }\end{array}$} & \multirow{2}{*}{$\begin{array}{l}\text { Cantidad de } \\
\text { participantes }\end{array}$} & \multicolumn{2}{|c|}{$\begin{array}{l}\text { Composición } \\
\text { por género }(\%)\end{array}$} & \multirow[t]{2}{*}{ Duración } \\
\hline & & & & & $\mathrm{F}$ & M & \\
\hline 1 & $\begin{array}{l}\text { Consecuencias de la } \\
\text { construcción del tranvía }\end{array}$ & $\begin{array}{l}\text { Espacio } \\
\text { público }\end{array}$ & $\begin{array}{l}\text { Sábado } 18 \text { de } \\
\text { marzo, 10:00 a.m. }\end{array}$ & $20 *$ & \multicolumn{2}{|c|}{ Indefinido } & $3: 45$ \\
\hline 2 & $\begin{array}{l}\text { Uso del espacio público } \\
\text { patrimonial }\end{array}$ & $\begin{array}{l}\text { Institución } \\
\text { autónoma }\end{array}$ & $\begin{array}{l}\text { Miércoles } 29 \text { de } \\
\text { marzo, 3:00 p.m. }\end{array}$ & 33 & 46 & 54 & $3: 10$ \\
\hline 3 & $\begin{array}{l}\text { La valoración del } \\
\text { patrimonio de Cuenca }\end{array}$ & $\begin{array}{l}\text { Institución } \\
\text { privada }\end{array}$ & $\begin{array}{l}\text { Martes } 11 \text { de abril, } \\
\text { 5:00 p.m. }\end{array}$ & 12 & 42 & 58 & $2: 45$ \\
\hline 4 & $\begin{array}{l}\text { Gobernanza participativa } \\
\text { en la gestión del centro } \\
\text { histórico de Cuenca }\end{array}$ & $\begin{array}{l}\text { Institución } \\
\text { educativa } \\
\text { pública }\end{array}$ & $\begin{array}{l}\text { Miércoles } 19 \text { de } \\
\text { abril, 3:00 p.m. }\end{array}$ & 40 & 40 & 60 & $3: 00$ \\
\hline
\end{tabular}

Fuente: Elaboración propia de la autora con datos obtenidos a partir de la ejecución de los foros híbridos en Cuenca, Ecuador.

limitación de conocimiento es necesaria para el éxito del foro ${ }^{63}$. Posteriormente, se generó la controversia en torno a un problema previamente identificado, que para el caso del primer foro fue la construcción de un tranvía que ha enfrentado a diversos actores ante las consecuencias negativas que el proyecto ha traído, no solo al centro histórico sino a toda la ciudad ${ }^{64}$. A partir de esta primera controversia identificada, se inició el primer debate exploratorio para determinar lo que los participantes consideraron que eran las problemáticas y necesidades más relevantes. Durante este proceso, varios actores del foro híbrido, a favor y en contra, expusieron sus ideas (Figura 1). Debido a la carga emocional de los debates, muchos argumentos tendieron a volverse personales, demostrando en este punto la importancia esencial del moderador y su capacidad para volver a encausar el debate hacia un terreno común. De este primer ejercicio se identificaron otras tres problemáticas importantes: la restricción del uso del espacio público patrimonial, la puesta en valor del centro histórico de Cuenca y la gobernanza participativa en la gestión del sitio Patrimonio Mundial, que fueron objeto de exploración en los siguientes tres foros híbridos y cuyos resultados se trabajarán, como ya se ha mencionado, en un artículo posterior.

La última parte de la ejecución de todos los foros híbridos consistió en demostrar a los participantes los límites del propio saber y, por tanto, la importancia de la generación de un conocimiento colaborativo. Para ello, se pasó a un formato de taller (workshop), donde, con la técnica del árbol ${ }^{65}$, se le pidió a los participantes -organizados en grupos- tratar de encontrar las causas y posibles soluciones a los problemas surgidos de la

63. Callon et al., Acting in an uncertain world.

64. Redacción Diario El Tiempo, «Frentistas del tranvía exigen culminación de obras», Diario El Tiempo, 12 de octubre de 2017, acceso: 07 de octubre de 2018, https://www.eltiempo.com.ec/noticias/cuenca/2/ frentistas-del-tranvia-exigen-culminacion-de-obras

65. Mayor información sobre esta técnica en: Gabriel Vergara Lara, La técnica del árbol para la toma de decisiones (México: Univalle, 2008): 350. 


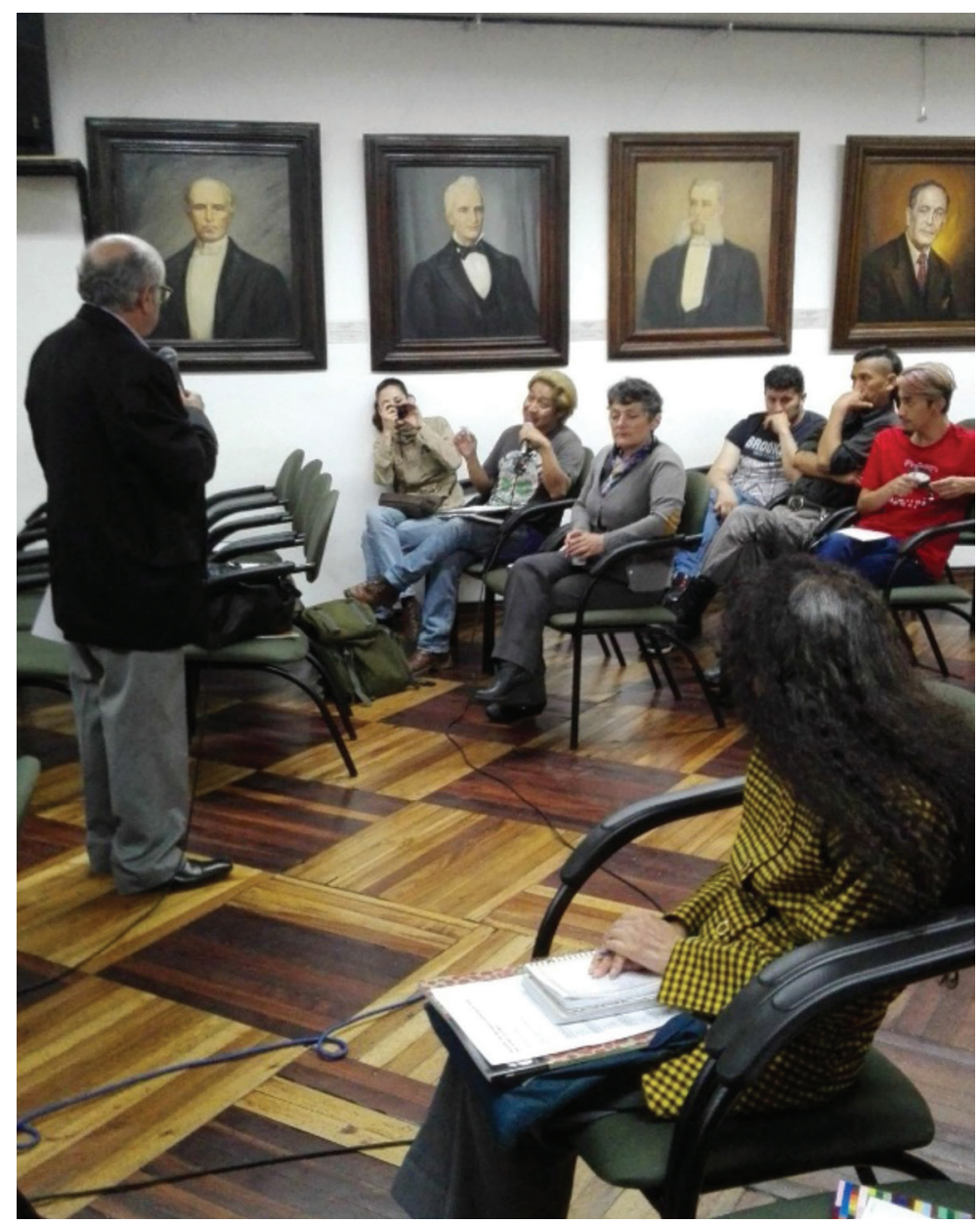

Discusión de controversias en el segundo foro híbrido. (Fotografía de la autora).

controversia (Figura 2). Este fue quizá el aspecto más enriquecedor de la realización de los foros híbridos porque, al tratar de explicar las posibles causas y consecuencias, los actores hicieron uso de sus propias percepciones, experticia y experiencia, para finalmente enfrentarse a las posturas de otros participantes y de este modo obligarse a buscar diferentes escenarios. La importancia en este sentido es que, incapaces de encontrar respuestas inmediatas, los integrantes de cada grupo se vieron obligados a oír las razones y explicaciones de los demás componentes del grupo. Al hacerlo, se dieron cuenta de la generación del conocimiento colaborativo, al ser capaces de visualizar claramente con cual información contaban y cual ignoraban.

Al final de cada taller se realizaron plenarias donde se expusieron las dificultades enfrentadas al momento de buscar soluciones. Al explicar las limitaciones del ejercicio, los participantes fueron capaces de entender que una socialización que produce consensos inmediatos, en realidad no es el resultado de un proceso colaborativo, sino más bien es 


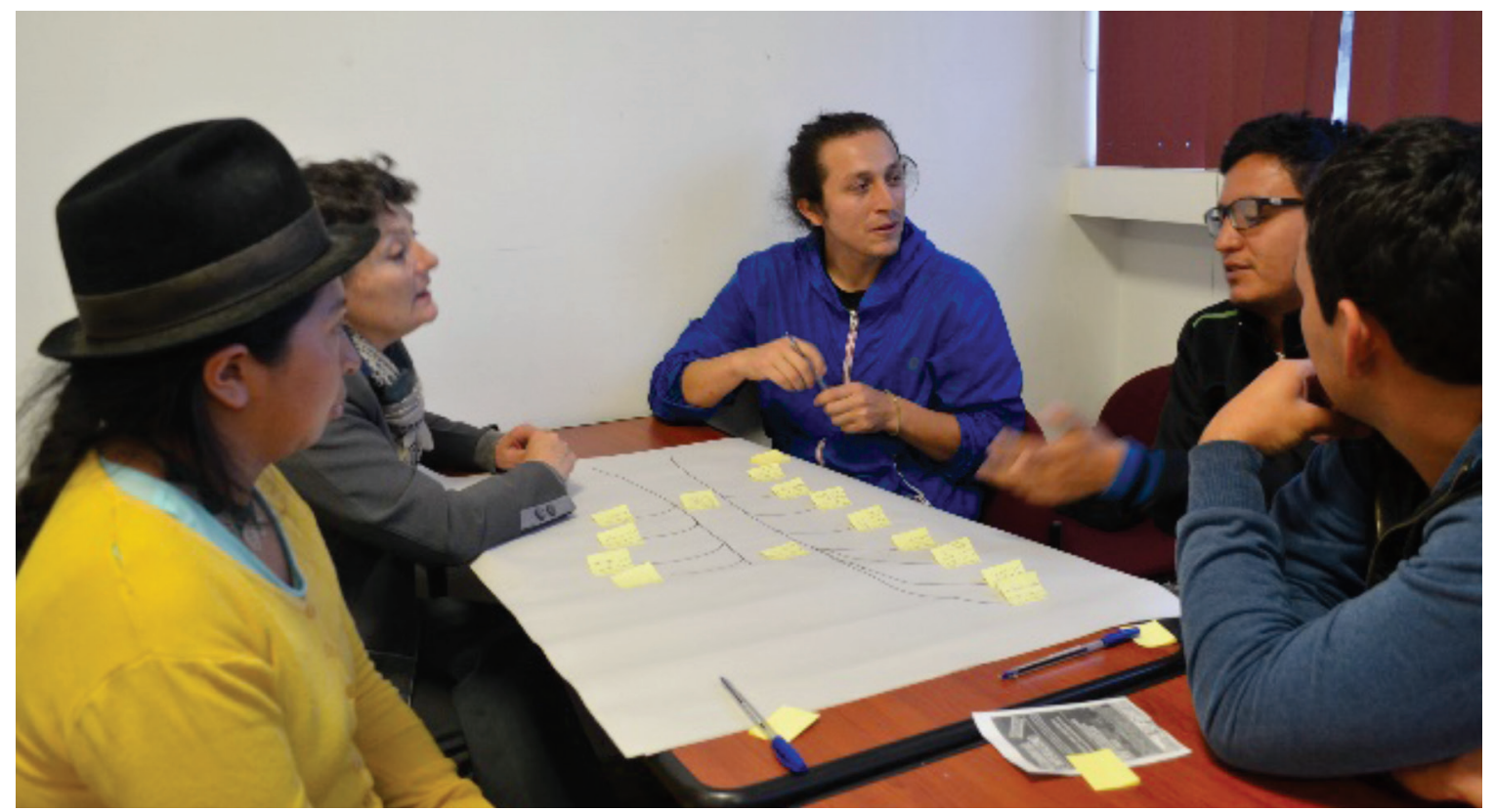

Participantes del tercer foro híbrido trabajando la técnica del árbol. Fotografía de la autora.

una forma de legitimar procesos políticos. De este modo, fueron conscientes de la importancia del conocimiento colaborativo como forma de empoderamiento porque les ayudó a comprender cómo debería funcionar la participación. En este sentido, la ciudadanía debe creer en el valor y dimensión del patrimonio para colaborar en los foros híbridos; pero, además, debe tener claro cuáles son los beneficios que espera obtener de este tipo de ejercicios participativos, más allá de cumplir con su deber cívico. Esto es necesario en la implicación y defensa de los postulados de los foros híbridos para conseguir la sostenibilidad. Así, la credibilidad y conocimiento del patrimonio y las expectativas reales sobre los beneficios que este pueda aportar, hará que la ciudadanía conozca y se reconozca en su patrimonio para que lo valore y defienda, lo que generaría sinergias que ayudarían a la continuidad de los foros híbridos.

\section{Discusión metodológica de la realización de los foros híbridos y su análisis contrastado}

La práctica metodológica de los foros híbridos en Cuenca ofrece una serie de datos que es importante compartir. Estos se refieren a las características de los participantes en relación con su género, área de conocimiento, composición etaria y otras características como su vinculación institucional a redes o libre ejercicio de la ciudadanía. Los datos, además, muestran la importante relación que los foros híbridos pueden llegar a tener con el espacio donde se desarrollan. La discusión sobre todos estos aspectos finalizará con una valoración de la aplicación metodológica en relación con los principios 
propuestos por Callon et al. ${ }^{66}$ y otras consideraciones que, de acuerdo con varios autores, han de cumplir los mecanismos de participación dentro de los parámetros que establece la sostenibilidad.

\section{Características de los participantes}

En el caso de los foros híbridos realizados en Cuenca, el análisis de los participantes muestra que, desde el punto de vista del género, los integrantes estuvieron relativamente equilibrados, con una pequeña superioridad del género masculino, como se aprecia en la tabla 1. Este resultado fue completamente casual; no obstante, es importante porque se repite en todos los foros realizados, lo que indica que, al menos desde el género, existe un relativo equilibrio en la voluntad de cooperar en este tipo de dispositivos de participación.

En lo que respecta a edades, estuvieron comprendidas entre los rangos de los 17 hasta los 70 años, siendo el intervalo de 20 a 30 y de 40 a 50 años los más representados en todos los foros híbridos. Esto demuestra que, en lo referente a la edad, faltaron adultos mayores y niños, lo que evidencia la necesidad de tener a representantes de todas las edades. Desde este punto, futuras investigaciones deberán determinar qué motiva a los diferentes grupos de edad a participar o no en este tipo de mecanismos, para tener una mejor idea de cómo llegarles.

En relación con el número de participantes y sus conocimientos, cada foro presentó componentes diferentes. En el primer foro se registró un promedio de 20 participantes, pues al tratarse de un espacio público abierto en determinados momentos durante su realización, el número aumentó, mientras que en otros disminuyó. El aumento y disminución de participantes se vio especialmente influenciado por la presencia de la Guardia Urbana Municipal, que cada determinado tiempo se detenía a solicitar permisos, cuestionando si se trataba de un evento o del libre ejercicio del derecho a la reunión. Una cuestión relevante que se produjo en este foro híbrido de manera más marcada que en otros, fue que los integrantes se identificaron ante todo como ciudadanos. Solo posteriormente, durante las intervenciones, algunos refirieron su profesión o trabajo para dar peso a su argumentación. En este primer foro se llevó como único registro un diario de campo, lo cual hizo difícil en la práctica llevar un conteo de los participantes de acuerdo con sus datos demográficos y socioculturales. Esta situación se corrigió en los siguientes foros con el uso de un registro que incluyó un apartado que informaba sobre los objetivos y fines del estudio, que además sirvió como consentimiento informado.

El segundo foro híbrido contó con 33 participantes registrados y fue el más heterogéneo de todos. Estuvo conformado por participantes con conocimientos múltiples en docencia, arte, fotografía, comunicación social, leyes, gestión, publicidad, turismo, ingeniería, psicología e historia. Esta vez, los integrantes del foro se presentaron primero como representantes de una determinada institución o profesionales, solo los que fueron como ciudadanos se presentaron en tales términos, haciendo siempre hincapié en su condición cívica. De estos, más de la mitad fueron representantes institucionales, con apenas

66. Callon et al., Acting in an uncertain world. 
un representante político y otros tres aspirantes a cargos públicos de tipo político. Lo que se pudo aprender de esto, es que en la realización de los foros híbridos en el espacio público, los participantes se identifican primero como ciudadanos, lo que establece una clara postura cívica común a todos que anula las jerarquías de manera inmediata. Sin embargo, en otros espacios se marca una clara diferencia entre profesionales, políticos y ciudadanos, haciendo difícil eliminar las jerarquías dadas por el poder político, el conocimiento académico o el carácter del espacio.

El tercer foro se realizó con un total de 12 personas, la mayor parte de ellas provenientes del ámbito de la cultura. En este caso, el debate fue mucho menos intenso, llegando en momentos a ser más una ratificación de una visión común ante los problemas discutidos. Esto, sin duda, demuestra que cuando el grupo es más homogéneo, el análisis de la controversia es menor, algo que se analizará de nuevo más adelante cuando se trate el tema del espacio en los foros híbridos.

Finalmente, en el cuarto foro participaron 40 personas. Su composición fue lo suficientemente heterogénea como para llevar a cabo el foro híbrido, a pesar de que la mayoría de participantes eran profesionales y estudiantes del ámbito de las humanidades. Los legos conformaron un cuarto del total de participantes (10 en total) y se contó con la presencia de dos políticos. En la siguiente tabla se detallan los datos de cada variable ofrecida en este apartado y otras relativas al tiempo de duración, horario y otras condiciones.

\section{Foros híbridos y su relación con el espacio}

La utilización de diferentes espacios para la ejecución de los foros híbridos proporcionó una serie de datos metodológicos relevantes que ayudan a comprender la importancia de los diversos escenarios para la realización de estos mecanismos y cómo afectan la participación. El primer foro híbrido se realizó el 18 de marzo de 2017 en una plaza pública dentro de los límites del centro histórico. Este ejercicio inicial se planeó de forma consciente fuera de un espacio institucional, pero trajo como consecuencia que ningún representante del gobierno local participara. Esta ausencia demuestra que, si bien se pueden ejecutar foros fuera de la institucionalidad, todavía es imprescindible realizar un esfuerzo especial para que la composición de grupo sea suficientemente equilibrada, incluyendo actores institucionales y políticos. En este contexto, el problema no es realmente el espacio público, sino la capacidad de convocatoria de los actores que se consigue únicamente a través del desarrollo de redes.

El segundo foro híbrido se realizó el 29 de marzo de 2017, en la Casa de la Cultura Ecuatoriana Núcleo del Azuay, una institución autónoma de gestión cultural que contribuye al desarrollo de los derechos culturales enmarcados en la política pública cultural del Estado ecuatoriano ${ }^{67}$. Este fue el foro híbrido con más éxito desde el punto de vista de la heterogeneidad, ya que incluyó tanto a representantes institucionales como políticos,

67. Ley Orgánica 23/2006 art. 3 del 3 de enero de 2006, de la Casa de la Cultura Ecuatoriana Benjamín Carrión (Registro Oficial 179). 
profesionales y actores externos. En contraste con el anterior, en este hubo una fuerte presencia de representantes institucionales y fue el que mayores tensiones generó entre los implicados, lo que evidenció más claramente las pugnas que existen entre las instituciones y los actores externos. Un hallazgo importante en este escenario fue que el nivel de intervención de los participantes externos se vio en algunos momentos minimizado por profesionales y autoridades que intentaban imponer su criterio. Sin embargo, cuando varios de los elementos institucionales se disponían a dejar el espacio después de su intervención, fueron recriminados por el grupo y acusados de utilizar el espacio como plataforma política. Cuando los integrantes se sienten apoyados como grupo, pueden evitar la manipulación política de este tipo de mecanismo de participación, que es quizá el mayor riesgo al que se enfrenta. Aun así, y pese a los esfuerzos del moderador, resultó difícil eliminar las jerarquías, pero quedó claro que el empoderamiento de los actores externos es clave para equilibrar el proceso.

El escenario del tercer foro híbrido fue el Museo de las Religiosas Conceptas, una institución privada perteneciente a la Iglesia católica. Se llevó a cabo el 11 de abril de 2017 y resultó peculiar por el nivel de participación y de controversia. Fue el que menos éxito de convocatoria tuvo, aun cuando se había llevado a cabo una amplia campaña de difusión por varios medios. El bajo nivel de participación hizo prácticamente innecesario el papel del moderador, especialmente porque el nivel de controversia fue también muy bajo. En este caso, aunque estuvieron presentes actores institucionales, no acudieron autoridades del gobierno, aun cuando se trata de una institución con acceso a canales de difusión y contactos institucionales. Sobre esta experiencia quedaron ciertas dudas, relacionadas con si la falta de controversia se debió a que, aun siendo un espacio privado, se trataba de un espacio vinculado a la sacralidad, o porque la composición del grupo no era totalmente heterogénea. Quizá la ausencia de políticos se produjo porque estos no están dispuestos o tienen menos interés en formar alianzas con cierto tipo de instituciones culturales o religiosas, o simplemente la escasa participación se produjo por la lluvia que cayó durante la tarde. Solo experiencias futuras lograrán responder a estas interrogantes.

El último foro híbrido se llevó a cabo el 19 de abril en la Universidad de Cuenca, institución educativa de carácter público. Aunque este foro evidenció, al igual que el segundo, que existe una tendencia a la imposición por parte de expertos y aquellos con capacidad de decisión, esto no limitó el nivel de participación y diálogo. Al comparar este foro con los otros, se piensa que en las condiciones adecuadas podría ser un buen espacio para realizar los foros híbridos de manera prolongada, gracias al alto poder de convocatoria que tienen las universidades, al menos en Cuenca, y porque - de entre todas las instituciones- es quizá la que mejores redes posee.

El análisis planteado en el párrafo anterior lleva a considerar que el espacio es un factor determinante que influencia la participación de los integrantes del foro híbrido. Los ejemplos expuestos demuestran que en diversos espacios las formas de participación son igualmente diferentes y que, además, su carácter (religioso, público, privado) también influye en la manera de intervenir de los participantes. En este sentido, el espacio se configura como un laboratorio que podría informar sobre las formas de participación y si estas son más o menos democráticas y equitativas dependiendo del escenario donde se desarrollen. Lo importante en cada caso es que el espacio cuente con una mínima infraestructura que garantice la ejecución de un foro híbrido de manera abierta y habilitada. 
En este apartado final es oportuno realizar una valoración de la metodología aplicada de los foros híbridos en función del cumplimiento de los principios de controversia, incertidumbre y precaución propuestos por Callon et al. ${ }^{68}$, que refieren a la discusión de diferentes posturas que permita considerar todos los posibles escenarios antes de plantear soluciones definitivas a una controversia. Del mismo modo, se cree que son importantes en la valoración de esta metodología, los criterios que diversos autores mantienen sobre las determinadas características que han de tener los mecanismos de participación, como son, en primer lugar, el nivel de implicación y la diversidad de los participantes ${ }^{69}$; el nivel de independencia de los actores externos respecto a los grupos de poder estable$\operatorname{cidos}^{70}$; y, por último, el nivel de apertura y equidad del mecanismo ${ }^{71}$. Los criterios de valoración aquí presentados (Tabla 2) ayudan a realizar una lectura que permita hacer una proyección a futuro y concluir si estos mecanismos tienen alguna posibilidad para la gestión sostenible del Sitio Patrimonio Mundial de Santa Ana de Cuenca a largo plazo.

Desde el punto de vista de la controversia generada, está claro que esta depende a su vez de la diversidad del grupo y sus conocimientos. En este sentido, algunos foros no resultaron lo suficientemente equilibrados, con lo cual es vital que, de seguir experimentando con esta metodología, se intente llegar a una mayor cantidad de agentes con conocimientos en disciplinas diferentes a las de las humanidades, que tendieron a ser dominantes. La mayor inclusión de conocimientos diversos es posible unicamente a través del desarrollo de redes ${ }^{72}$ que en el caso de Cuenca fueron pocas y débiles. En este sentido, los foros híbridos mostraron que, cuando los participantes se compromenten, se puede comenzar a formar redes ya que, al basarse en la confianza generada a partir de la participación y colaboración, los actores pueden generar un tejido que se prologa hacia una diversidad de ámbitos.

En lo que respecta a la incertidumbre y la precaución, los resultados de los diversos foros híbridos demostraron que al exponer a los integrantes a situaciones que no pueden explicar, estos se vieron obligados a escuchar propuestas y considerar diversos escenarios. En este punto, los participantes fueron concientes de que el mantenimiento de determinadas posturas no era posible si de lo que se trataba era de dar una solución real a una controversia real. Aquí la capacidad de réplica argumentada minimiza la visión de túnel

68. Callon et al., Acting in an uncertain world.

69. Christina Aas, Adele Ladkin y John Fletcher, «Stakeholder Collaboration and Heritage Management», Annals of Tourism Research, 32-1 (2005): 28-48. Lusiani et al., «The Complexity of Becoming: Collaborative Planning and Cultural Heritage», Journal of Cultural Heritage Management and Sustainable Development 3-2 (2013): 148-62. S. Mostafa Rasoolimanesh et al., «Factors Influencing Residents' Perceptions toward Tourism Development: Differences across Rural and Urban World Heritage Sites», Journal of Travel Research 56, n. ${ }^{\circ} 6$ (julio 17, 2017): 760-75. Arnaboldi y Spiller, «Actor-Network...».

70. René Kemp, Saeed Parto, y Robert B Gibson, «Governance for Sustainable Development: Moving from Theory to Practice», International Journal of Sustainable Development 8, n. ${ }^{\circ}$ 1-2 (2005): 12-30. Landorf, «Governance in Historic...». Ripp y Rodwell, «Governance in UNESCO...».

71. Farías, «Devising Hybrid Forums...».

72. Latour, «Reassembling the Social». 
y pone en marcha un sinfín de posibilidades no consideradas. El problema en este sentido se produjo con los representantes institucionales, ya que estos son portavoces de determinadas posturas y no de las propias, lo que les hace el componente más resistente. Por ello, es indispensable que los participantes de este tipo no superen a los actores externos independientes y, por tanto, de que quien organiza el foro híbrido ha de asegurarse de que el grupo esté conformado de manera equitativa, lo cual no se refiere a un número particular de implicados sino a la no monopolización del debate. En este contexto, los foros híbridos realizados en Cuenca mostraron que en la práctica no es posible llevar a cabo este tipo de participación sin un mediador.

Después de valorar los resultados con base en el criterio de diversos autores, se puede afirmar que los foros híbridos tienen grandes posibilidades de conformarse como herramientas metodológicas para la gestión sostenible de lugares patrimoniales como es el caso del Sitio Patrimonio Mundial de Cuenca a largo plazo. Sin embargo, es necesario que los actores trabajen en el desarrollo de redes que permitan una mayor circulación de información y, sobre todo, que permita una mayor vinculación de actores diversos para generar empoderamiento. Solo así se conseguirá un mayor poder de convocatoria desde todos los sectores que evite la manipulación institucional del mecanismo de participación, que garantice la equitatividad en la participación y, por lo tanto, la continuidad del foro híbrido y su activación cuando las circunstancias así lo exijan.

TABLA 2

Criterios para la valoración de los foros híbridos

\begin{tabular}{lcccc}
\hline \multicolumn{1}{c}{ Foro } & 1 & 2 & 3 & 4 \\
Nivel de diversidad & medio & alto & bajo & alto \\
Nivel de apertura & alto & alto & alto & alto \\
Nivel de participación & alto & medio & alto & medio \\
Nivel de controversia & alto & alto & bajo & alto \\
Nivel de incertidumbre & alto & alto & alto & alto \\
Nivel de precaución & alto & alto & alto & alto \\
Nivel de independencia de la participación & alto & medio & alto & alto \\
\hline
\end{tabular}

Fuente: Elaboración propia de la autora con datos obtenidos a partir de la ejecución de los foros híbridos en Cuenca, Ecuador.

\section{Conclusiones}

Después de haber analizado la literatura académica sobre la importancia de la gobernanza participativa en la gestión de las Ciudades Históricas Patrimonio Mundial, se concluye que para materializar en la práctica los supuestos teóricos sobre los beneficios que esta supone, es necesario desarrollar metodologías participativas. En este contexto, este artículo ha propuesto la metodología de los denominados foros híbridos ${ }^{73}$ como una contribución a las opciones metodológicas para la gestión participativa de los Centros Históricos Patrimonio Mundial. Como se ha señalado, los foros híbridos

73. Callon et al., Acting in an uncertain world. 
son instrumentos para la exploración de la relación entre los diversos actores y el patrimonio, pero también son una herramienta de empoderamiento porque permite a estos, a través de la construcción de un conocimiento colaborativo desde diferentes posturas y disciplinas, buscar soluciones diversas a las controversias que se puedan generar en torno al patrimonio.

Para comprobar la utilidad de los foros híbridos en la práctica, este estudio ha expuesto el proceso metodológico necesario para la ejecución de estos en la realidad, tomando como ejemplo el caso del Centro Histórico Patrimonio Mundial de Cuenca, en Ecuador. La discusión metodológica de las experiencias de los cuatro foros híbridos realizados lleva a concluir una serie de cuestiones. La primera es que la heterogeneidad de participantes es indispensable en el proceso, ya que sin esta no se pueden cumplir los principios de controversia, incertidumbre, precaución y continuidad aquí propuestos. Una segunda cuestión de las conclusiones es que los mecanismos de participación necesitan de la existencia de redes colaborativas entre actores para garantizar su continuidad. En este sentido se ha visto que los foros híbridos ofrecen una gran posibilidad para su desarrollo, pues el diálogo franco y abierto entre diversos implicados en un ambiente sin jerarquías, desarrolla la confianza entre ellos. Otra cuestión relevante es el espacio, aquí se ha argumentado, por una parte, que se necesita una mínima infraestructura y formalismo para que los foros se puedan realizar de manera abierta y habilitada; y por otra parte, que los diferentes escenarios dan como resultado diferentes formas de aproximación a las controversias. Esto es positivo porque el espacio se conforma como un laboratorio, donde los datos producidos informan sobre actitudes y posturas entre participantes y además pueden ayudar de gran manera a corregir cuestiones dentro del proceso en tanto se trata de una metodología experimental. En el caso del centro histórico de Cuenca, se ha visto que la escasa existencia de redes entre actores, el limitado acceso a la información institucional y la ausencia de participantes políticos, son cuestiones que se deben trabajar justamente desde las posibilidades que ofrecen los foros híbridos a través de la construcción de conocimiento colaborativo.

En lo que respecta a si la metodología de los foros híbridos es válida como herramienta para la gestión participativa de las Ciudades Históricas Patrimonio Mundial a largo plazo, se concluye que los foros híbridos se proyectan como una interesante metodología que fortalece las relaciones entre los actores y el patrimonio a través del empoderamiento. Esto permite una acción conjunta indispensable para la continuidad de una ciudad Patrimonio Mundial en el futuro. Sin embargo, la clave está en que los actores sepan cuál será el beneficio de invertir su tiempo en estos procesos a largo plazo. La iniciativa civil es importante, pero la retribución por el civismo también es necesaria. Con base en los argumentos aquí propuestos, se puede afirmar que para lograr la continuidad del patrimonio y el bien común es necesario prestar atención a lo que los gobernantes, gestores y ciudadanos tienen que decir, no solo en un momento determinado, sino de manera permanente. De ahí que cada foro híbrido presenta la oportunidad de conocer sobre las necesidades, aspiraciones y expectativas que los diversos participantes tienen en relación con un Sitio Patrimonio Mundial. Esto implica un valor considerable para la gestión participativa, pues las Ciudades Históricas Patrimonio Mundial no son espacios vacuos, sino todo lo contrario, dependen directamente de aquellos que viven y se relacionan 
directamente con ellas. Si desde la gestión patrimonial estas relaciones no son claras, entonces no se puede hablar de gestión sostenible de las ciudades Patrimonio Mundial.

\title{
ABSTRACT \\ Sustainable participatory management of the world heritage through hybrid forums. The case of the historic center of Santa Ana de Cuenca
}

\begin{abstract}
Currently, participatory management is deemed to be a key element in the sustainable development agenda. It is within this context that UNESCO has sought to incorporate sustainability in the management of world heritage cities. Nevertheless, these efforts have been constricted by a lack of proper methodologies that allow for participatory management of the heritage according to each context. Consequently, the use of the so-called hybrid forums is put forth as a participation mechanism. The world heritage site of Cuenca, Ecuador, was the focus of the case study that is presented here. The generated data will serve as a base to determine if this methodology is effective for sustainable management of the world heritage in the long run.
\end{abstract}

Key words: Methodology, hybrid forums, sustainability, participatory management, world heritage.

\section{RÉSUMÉ}

La gestion participative et durable du patrimoine mondial à travers des forums hybrides. Le cas du centre historique de Santa Ana de Cuenca

De nos jours, la gouvernance participative est considérée comme un sujet clé dans les agendas pour le développement durable. Dans ce contexte, l'UNESCO a visé à intégrer la durabilité à la gestion des villes historiques patrimoine mondial. Cependant, ces efforts ont été limités à cause du manque des méthodologies permettant la gestion participative du patrimoine d'après les caractéristiques de chaque contexte. De ce fait, l'utilisation des forums hybrides est proposée comme moyen de participation partant du cas d'étude de la ville patrimoine mondial de Cuenca en Équateur. À partir des données, on cherche établir si cette méthodologie est effective pour la gestion durable du patrimoine mondial à long terme.

Mots-clés: Méthodologie, forums hybrides, durabilité, gouvernance participative, patrimoine mondial.

\section{Bibliografía}

Aas, Christina, Adele Ladkin y John Fletcher. «Stakeholder Collaboration and Heritage Management». Annals of Tourism Research 32, n. ${ }^{\circ} 1$ (2005): 28-48. doi:10.1016/j.annals.2004.04.005.

Arnaboldi, Michela y Nicola Spiller. «Actor-Network Theory and Stakeholder Collaboration: The Case of Cultural Districts». Tourism Management 32, n. ${ }^{\circ} 3$ (2011): 641-54. doi:10.1016/j.tourman.2010.05.016.

Arnstein, Sherry. «A ladder of citizen participation». Journal of the American Institute of planners, 35-4, (1969): 216-224.

Avritzer, Leonardo. «Democracy and the Public Space in Latin America», 2009. doi:10.1515/9781400825011. 
Bandarin, Francesco y Ron van Oers, eds. Reconnecting the City, Oxford, UK: John Wiley \& Sons, Ltd., 2014, doi:10.1002/9781118383940

Bevir, Mark. A theory of governance. Berkeley/Los Angeles, California: University of California Press, 2013.

Bossel, Hartmut. Indicators for Sustainable Development: Theory, Method, Applications International Institute for Sustainable Development Winnipeg, 1999.

Brundtland, Gro Harlem. «Our common future». Report of the World Commission on environment and development: United Nations, 1987.

Callon, Michael, Pierre Lascoumes y Yannick Barthe. Acting in an Uncertain World: An Essay on Technical Democracy. MIT press, 2009.

Carrión, Fernando. «El centro histórico como proyecto y objeto de deseo». EURE (Santiago) 31, n. ${ }^{\circ} 93$ (2005). doi:10.4067/s0250-71612005009300006.

Carrión, Fernando. «Centro Histórico: La polisemia del espacio público», Redalyc.Org,2008. Acceso: 07 de octubre, 2018. http://www.redalyc.org/pdf/1151/115112535008.pdf

Chhotray, Vasudha y Gerry Stoker. Governance Theory and Practice: A Cross Disciplinary Approach. Basingstoke, Palgrave Macmillan, 2009.

Condon, Patrick M. Design Charrettes for Sustainable Communities. Island Press, 2012.

Consejo de Europa (2005). Serie de Tratados del Consejo de Europa n. ${ }^{\circ} 199$, 2005, https://rm.coe.int/16806a18d3

González, Alfonso. «El turismo desde un enfoque de sociología constructivista». Teoría y Praxis 5, n. ${ }^{\circ} 6$ (2009): 109-24. doi:10.22403/uqroomx/typ06/06.

Díaz García, Paloma. «El principio de precaución como base de los foros híbridos». Ética en la práctica. Granada: Universidad de Granada, 2008.

Evans, Peter. «Development as Institutional Change: The Pitfalls of Monocropping and the Potentials of Deliberation». Studies in Comparative International Development 38, n. ${ }^{\circ} 4$ (2004). doi:10.1007/bf02686327.

Farías, Ignacio. «Devising Hybrid Forums». City 20, n. ${ }^{\circ} 4$ (marzo 2016): 549-62. doi: 10.1080/13604813.2016.1193998.

Fischer, Frank. «Participatory Governance: From Theory To Practice». Oxford Handbooks Online, 2012. doi:10.1093/oxfordhb/9780199560530.013.0032.

Galla, Amareswar. World Heritage: Benefits Beyond Borders. Cambridge University Press, 2012.

Gallent, Nick. «Strategic-Local Tensions and the Spatial Planning Approach in England». Planning Theory \& Practice, 2008. doi:10.1080/14649350802277795.

Goldfrank, Benjamin. «The Fragile Flower of Local Democracy: a Case Study of Decentralization/Participation in Montevideo». Politics \& Society, 2002. doi:10.1177/0032329202030001003.

Goldfrank, Benjamin. «Lessons from Latin America's experience with participatory budgeting». Participatory budgeting, editado por Awar Shah, 91-126. Washington, DC: World Bank, 2007.

Goldfrank, Benjamin. Deepening Local Democracy in Latin America: Participation, Decentralization, and the Left. The Pennsylvania State University Press, 2011.

Harrison, Rodney. Heritage: Critical Approaches. Routledge, 2013.

Kemp, René, Saeed Parto y Robert B. Gibson. «Governance for Sustainable Development: Moving from Theory to Practice». International Journal of Sustainable Development 8, n. ${ }^{\circ} 1-2,2005$. https://doi.org/10.1504/ IJSD.2005.007372 
Labadi, Sofia y William Logan. «Urban heritage, development and sustainability». Londres/New York, Routledge, 2015.

Landorf, Chiristine. «Governance in historic urban environments: A theoretical review». International Journal of Heritage and Sustainable Development, 1(1), 2011.

Latour, Bruno. «On actor-network theory: A few clarifications». Soziale welt, 1996.

Latour, Bruno. Reassembling the Social. Hampshire. Oxford University Press, 2007.

Lawton, Laura J. y David B. Weaver. «Using Residents' Perceptions Research to Inform Planning and Management for Sustainable Tourism: A Study of the Gold Coast Schoolies Week, a Contentious Tourism Event». Journal of Sustainable Tourism 23-5, May 28, 2015. https://doi.org/10.1080/09669582.2014.991 398

Li, Yulong y Caroline Hunter. «Community Involvement for Sustainable Heritage Tourism: A Conceptual Model». Journal of Cultural Heritage Management and Sustainable Development 5, n. ${ }^{\circ}$ 3, 2015. https://doi. org/10.1108/JCHMSD-08-2014-0027

Lusiani et al., "The Complexity of Becoming: Collaborative Planning and Cultural Heritage», Journal of Cultural Heritage Management and Sustainable Development 3-2, 2013. https://doi.org/10.1108/ JCHMSD-10-2012-0053

Makuvaza, Simon. The Management of Cultural World Heritage Sites and Development in Africa: History, Nomination Processes and Representation on the World Heritage List. Springer Science \& Business Media, 2014.

Makuvaza, Simon. Aspects of Management Planning for Cultural World Heritage Sites Principles, Approaches and Practices. Cham: Springer International Publishing, 2018.

Molina, Bárbara. «La incorporación de la cultura y el patrimonio en el desarrollo sostenible: desafíos y posibilidades». Humanidades 8, n. ${ }^{\circ}$ 1, enero 2018. doi:10.15517/h.v8i1.31465.

Monteiro, Vasco, Marco Painho y Eric Vaz. «Is the Heritage Really Important? A Theoretical Framework for Heritage Reputation Using Citizen Sensing». Habitat International 45, 2015. doi:10.1016/j. habitatint.2014.06.022.

ONU. Agenda 21, Programa de Acción las Naciones Unidas. New York: UN Publications, 1992.

Programa de las Naciones Unidas para el Desarrollo (PNUD). Agenda para el Desarrollo Sostenible 2030, 2016.

Roders, Ana Pereira y Ron Van Oers. «World Heritage Cities Management». Facilities 29, n. ${ }^{\circ}$ 7/8, 2011. doi:10.1108/02632771111130898.

Rob Pickard. «Management strategies for historic towns in Europe». Urban heritage, development and sustainability: International frameworks, national and local governance, editado por Sofia Labadi. London/New York: Routledge, 2016.

Rasoolimanesh, S. Mostafa, Mastura Jaafar, A. Ghafar Ahmad y Rabeeh Barghi. «Community Participation in World Heritage Site Conservation and Tourism Development.» Tourism Management 58, 2017. doi:10.1016/j.tourman.2016.10.016.

Rey Pérez, Julia et al. Paisaje urbano histórico. La aplicación de la recomendación sobre el paisaje urbano histórico (PUH) en Cuenca (Ecuador). Una nueva aproximación al patrimonio cultural y natural. Universidad de Cuenca, Cuenca, 2017.

Ringbeck, Birgitta. «Management Plans for World Heritage Sites». A Practical Guide, 2008.

Ripp, Matthias y Dennis Rodwell. "Governance in UNESCO World Heritage Sites: Reframing the Role of Management Plans as a Tool to Improve Community Engagement». Aspects of Management Planning for Cultural World Heritage Sites, 2017. doi:10.1007/978-3-319-69856-4_18. 
Rocha, Elizabeth M. «A Ladder of Empowerment», Journal of Planning Education and Research 17, n. ${ }^{\circ}$ 1, septiembre 25, 1997. https://doi.org/10.1177/0739456X9701700104

Sen, Amartya. «Development as Capability Expansion». Human Development and the International Development Strategy for the 1990s, 1990. doi:10.1007/978-1-349-21136-4_3.

Schneider, Hartmut. «Participatory Governance for Poverty Reduction». Journal of International Development 11, n. ${ }^{\circ}$ 4, 1999. doi:10.1002/(sici)1099-1328(199906)11:4<521::aid-jid599>3.0.co;2-j.

Smith, Laurajane. Uses of Heritage. London: Routlegde, 2006.

Soini, Katriina y Joost Dessein. «Culture-Sustainability Relation: Towards a Conceptual Framework». Sustainability 8 , n. ${ }^{\circ}$ 2, noviembre 2016. doi:10.3390/su8020167.

Speer, Johanna. «Participatory Governance Reform: A Good Strategy for Increasing Government Responsiveness and Improving Public Services?» World Development 40, n. ${ }^{\circ}$ 12, 2012. doi:10.1016/j. worlddev.2012.05.034

The World Bank. «Participatory Development and the World Bank». World Bank Discussion Papers, Washington, DC, 1992. doi:10.1596/0-8213-2249-4.

UNESCO. Convención sobre la Protección del Patrimonio Mundial Cultural y Natural, 1972. Acceso: 07 de octubre, 2018. http://portal.unesco.org/es/ev.phpURL_ID=13055\&URL_DO=DO_TOPIC\&URL_ SECTION=201.html

UNESCO. Convención para la salvaguardia del Patrimonio Cultural Inmaterial, 2003. Acceso: 07 de octubre, 2018. http://unesdoc.unesco.org/images/0013/001325/132540s.pdf

UNESCO. Convención sobre la protección y promoción de la diversidad de las expresiones culturales, 2005. Acceso: 07 de octubre, 2018. http://unesdoc.unesco.org/images/0014/001429/142919s.pdf

UNESCO. Recomendación sobre el paisaje urbano histórico, con inclusión de un glosario de definiciones, 2011. Acceso: 07 de octubre, 2018. http://portal.unesco.org/es/ev.php-URL_ID=48857\&URL_DO=DO_ TOPIC\&URL_SECTION=201.html

UNESCO. Directrices Operativas para la aplicación de la Convención de Patrimonio Mundial, 2017. Acceso: 07 de octubre, 2018. https://whc.unesco.org/en/guidelines/

Vergara Lara, Gabriel. La técnica del árbol para la toma de decisiones. México: Univalle, 2008.

Vlassis, Antonios. «Culture in the Post-2015 Development Agenda: the Anatomy of an International Mobilisation». Third World Quarterly 36, n. ${ }^{\circ}$ 9, febrero 2015. doi:10.1080/01436597.2015.1052064.

Warren, Mark E. «Institutionalizing Deliberative Democracy». Deliberation, Participation and Democracy: Can the People Govern? London and New York: Palgrave Macmillan, 2007. doi:10.1057/9780230591080_13.

Wilcox, David. «The guide to effective participation». Delta Press, 1994.

Williams, Paul. «The Governance of Sustainable Development in Wales». Local Environment 11, n. ${ }^{\circ}$ 3, 2006. doi:10.1080/13549830600558754.

Yung, Esther H.k. y Edwin H.w. Chan. «Problem Issues of Public Participation in Built-Heritage Conservation: Two Controversial Cases in Hong Kong». Habitat International 35, n. ${ }^{\circ}$ 3, 2011. doi:10.1016/j. habitatint.2010.12.004. 